\title{
Observational study of a patient and doctor directed pre-referral questionnaire for an early arthritis clinic
}

\author{
Uta Arndt • Frank Behrens · Hans Rudolf Ziswiler • \\ Joachim Peter Kaltwasser · Burkhard Möller
}

Received: 1 February 2007 / Accepted: 22 May 2007 / Published online: 15 June 2007

(C) Springer-Verlag 2007

\begin{abstract}
We evaluated a combined physician and patient questionnaire designed for identifying early rheumatoid arthritis (RA) and spondyloarthritis ( $\mathrm{SpA}$ ) in a cohort of 220 patients supposed for admission to an early arthritis clinic (EAC). The documents including personal and basis demographic data, referral diagnosis, questions related to RA and SpA classification criteria, functional limitations and previous diagnostic and therapeutic attempts were faxtransmitted to referring practices and returned before first EAC appointment. 125 referrals before introduction of the questionnaire served as controls. We found that a functional impairment of the hands provided more accurate prediction of RA than reports on morning stiffness or joint swelling. No clinical data proved predictive for SpA. We observed an unintended increase in the prescription of analgesics/ NSAID and corticosteroids. In conclusion, questionnaires as designed here may provide substantial information for diagnosis of RA, but also imply the risk of unmeant therapeutic attempts.
\end{abstract}

Keywords Early rheumatoid arthritis · Spondyloarthritis . Questionnaire

U. Arndt $\cdot$ F. Behrens $\cdot$ J. P. Kaltwasser

Medical Department II, Centre for Rheumatic Diseases,

J.W. Goethe-University, Frankfurt, Germany

H. R. Ziswiler · B. Möller ( $\square)$

Department for Rheumatology and Clinical

Immunology/Allergology,

Inselspital-University Hospital, 3010 Berne, Switzerland

e-mail: burkhard.moeller@insel.ch

\section{Introduction}

Early diagnosis of inflammatory arthritis (IA), especially $\mathrm{RA}$ and $\mathrm{SpA}$ belong to the most important challenges in rheumatology. The goal for rapid start of treatment in early RA is clearly formulated [1], in similar suggested for SpA [2], and the early treatment paradigm appears accepted in the meantime [3]. Existing early referral recommendations might provide a useful tool for primary care physicians in the management of early RA patients [4]. Realization of these recommendations, however, appears more difficult than expected from a rheumatologist's point of view [5-7]. Many referrals to EACs are inappropriate despite implemented education efforts [5], and it consumes substantial resources of an EAC to exclude inflammatory arthritis in about $50 \%$ of referred cases. In order to design an advance information instrument for triaging, we drafted a short questionnaire for referring doctors, which included a patient directed section.

\section{Patients and methods}

Data were analyzed from 345 admissions to an EAC at Frankfurt University Hospital, Germany. A total of 220 patients were referred within 12 months after introduction of the questionnaire, and 125 referrals before introduction served as control cases. Hardcopy documents were transmitted by fax to the referring doctor's practice, and also returned by fax when completed. The questionnaire (Table 1) was primarily designed to cover the ACR classification criteria for RA (questions P1, Q2, and Q5 [8]), the criterion of inflammatory back pain in its original version (questions P1, P2, Q3 in context with age and symptom duration [9]), and the ESSG criteria for the diagnosis of 
Table 1 Patients and physicians directed questionnaire
Questions marked with a bold dot had to be answered in plain text. Categorical items were discriminated for positive, negative, or not done

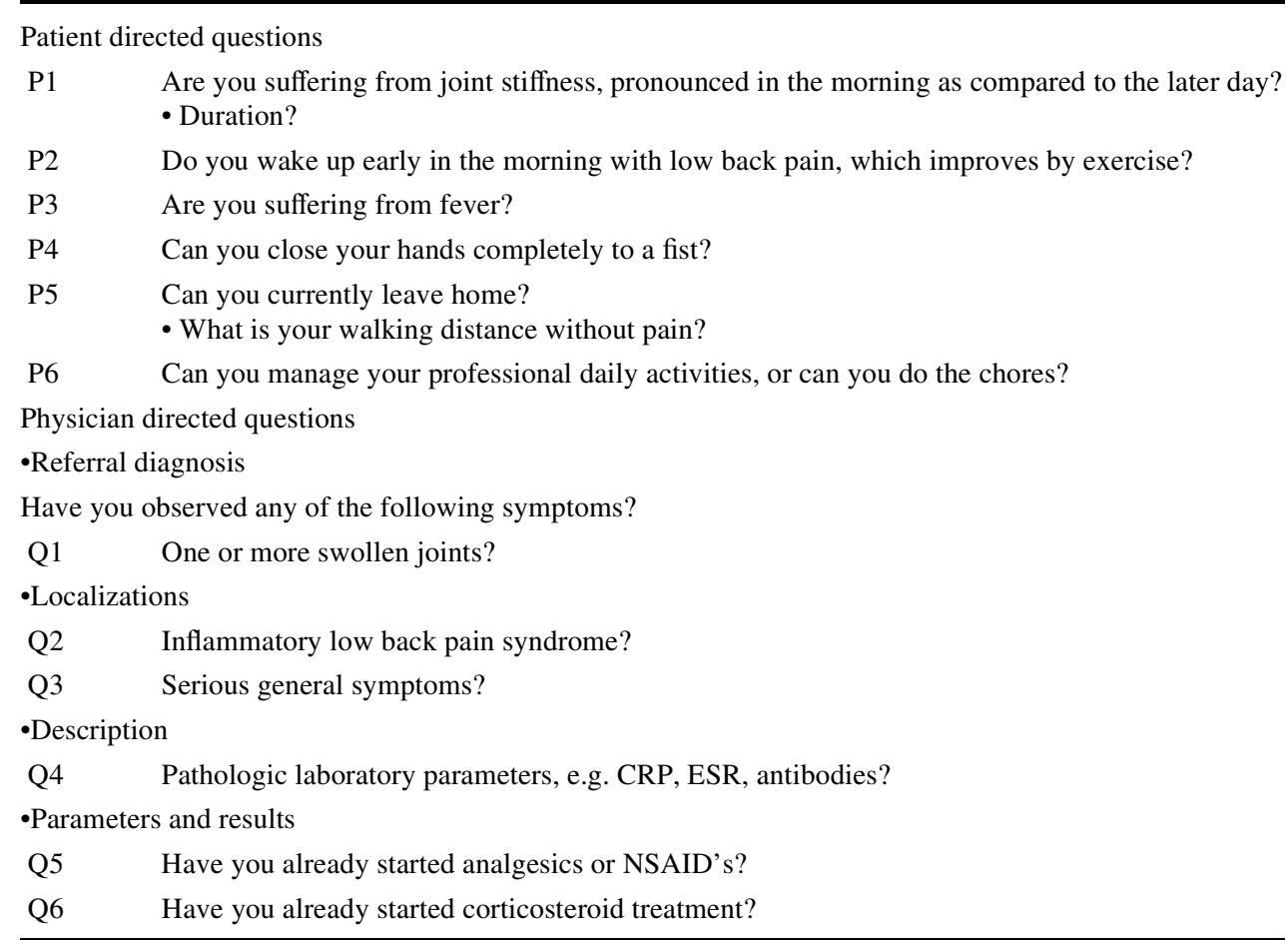

spondylarthropathy [10]. We additionally asked for signs of serious general symptoms (P3, Q4), important functional limitations (P4-P6), laboratory data (Q5), and previous therapeutic attempts (Q6-Q7). In order to avoid any ethical issue and to see all proposed patients, complete response to the questionnaire items was gently asked for, but not a prerequisite for accepting the referral. Age, gender, and symptom duration had to be given by the patients. Referral diagnoses, detailed information on involved joints, laboratory and previous therapeutic efforts had to be filled in plain text fields by the referring colleagues. For more details see a translated version in Table 1.

EAC diagnoses were done at first two consultations by two board certified rheumatologists (BM and PK). First appointment included the review of the referral documents, taking the patient history, a physical examination, and ordering of necessary additional diagnostic procedures. Patients were diagnosed according to the international classification of diseases (ICD-10). Referral and EAC diagnoses were then grouped into eight cohorts: RA according to the ACR classification criteria [8], suspected RA, not yet fulfilling ACR criteria, SpA according to the ESSG criteria [10], definitive diagnosis of other types of inflammatory arthritis (IA, undifferentiated mono- or oligoarthritis, psoriatic arthritis not fulfilling ESSG criteria, crystal induced arthritides), osteoarthritis (OA) and degenerative joint diseases, inflammatory connective tissue diseases and vasculitides (CTD), other definitive (tendopathies, regional pain syndromes, hypermobility, fibromyalgia), and unclear conditions. Statistics were done using SPSS version 13.1 by calculation of likelihood ratios (LR) and binary stepwise logistic regression analyzes (ANOVA). The degree of freedom $(d f)$ reflects the number of groups in calculation of LR $\left[n=\left(n_{\text {vert }}-1\right)\left(n_{\text {horiz }}-1\right)\right]$.

\section{Results}

The frequency of reported items varied from $94.5 \%$ for the mandatory referral diagnosis to $63.3 \%$ for laboratory parameters, $50.5 \%$ for clinical data including the joint swelling status, and $52 \%$ for the completed patient questionnaire. Results or original films of conventional X-ray were provided for $36 \%$ and of magnetic resonance imaging (MRI) or computed X-ray (CT) for $19.6 \%$ of the cases.

Despite significant statistical accordance of referral and EAC diagnoses $(\mathrm{LR}=199.4, n=331, d f=42, P<0.001)$, relevant differences had to be stated (Table 2). RA appeared overestimated and $\mathrm{SpA}$ underestimated in their prevalence among the referral diagnoses, and non-inflammatory conditions were frequently misdiagnosed as inflammatory entities. A substantial number of patients with RA referral diagnosis could be also immediately classified as inflammatory connective tissue disorders due to present, but undetected or misinterpreted symptoms. Notably, 12 of 22 RA, 4 of $10 \mathrm{SpA}, 19$ of $29 \mathrm{IA}$, and approximately $58 \%$ of all referred patients had symptom duration of more than 1 year. 
Table 2 Matrix of referral diagnoses (vertical) given in absolute numbers, and rheumatologist diagnoses (horizontal) defined latest after second appointment in the early arthritis clinic

Rheumatologists diagnoses

RA RA SpA Other OA CTD Miscell. Uncertain Total susp. IA

\begin{tabular}{lrrrrrrrrr}
\hline \multicolumn{7}{l}{ Referral diagnoses } \\
RA & 12 & 1 & 0 & 1 & 0 & 10 & 1 & 0 & 25 \\
RA susp. & 8 & 10 & 0 & 5 & 17 & 1 & 16 & 30 & 87 \\
SpA & 0 & 0 & 4 & 1 & 0 & 0 & 0 & 1 & 6 \\
Other IA & 0 & 0 & 2 & 7 & 0 & 0 & 1 & 0 & 10 \\
OA & 0 & 0 & 1 & 0 & 2 & 0 & 0 & 0 & 3 \\
CTD & 0 & 0 & 0 & 0 & 0 & 0 & 0 & 0 & 0 \\
Miscell. & 0 & 0 & 0 & 0 & 1 & 1 & 2 & 1 & 5 \\
Uncertain & 2 & 1 & 7 & 22 & 20 & 30 & 44 & 69 & 195 \\
Total & 22 & 12 & 14 & 37 & 40 & 41 & 64 & 101 & 331 \\
\hline
\end{tabular}

There is significant overall agreement, however, suspected RA changed to OA in 17, and definitive RA to CTD (predominantly to Sjoegren syndrome) in ten patients, whilst SpA appeared underrepresented among the referral diagnoses

$R A$, Rheumatoid arthritis; $S p A$, spondyloarthritis; $I A$, other inflammatory arthritis not fulfilling RA or SpA criteria, among them psoriatic $(n=8)$ and crystal induced arthritis $(n=4) ; O A$, osteoarthritis; CTD, inflammatory connective tissue diseases, miscellaneous (including enthesitis, tendonitis, regional or general pain syndromes)

\section{Prediction of RA}

Valuable information for RA diagnosis could be obtained from the questionnaires (Table 3): reporting of any joint swelling was associated with the referral diagnosis of RA or suspected RA (LR 8.16, $n=111, d f=1, P=0.004$ ), and with definitive EAC diagnosis of RA ( $\mathrm{LR}=9.20, n=111$, $d f=1, P=0.002)$. Following the reports, swollen joints ( $n=66)$ were predominantly localized at the hands $(n=45)$ or knee $(n=12)$. However, restriction of the swollen joint status to localisations at hands or fingers was not predictive for RA diagnosis at EAC, nor did this information significantly coincide with a definitive or tentative RA referral diagnosis. This result was due to the fact that diagnoses had to be revised to $\mathrm{OA}$ in seven, to arthritis other than RA in five, to inflammatory CTD in two patients. In addition, synovitis could not be objectified in 21 other of the referred patients, thereby forestalling confirmation of suspected RA. Of note, the patient information on morning stiffness was neither predictive for referral nor EAC RA diagnoses. In contrast, information about limitations when clenching the hands completely to a fist could be associated with RA referral diagnoses (definitive: $\mathrm{LR}=6.11, n=105, d f=1$, $P=0.013$, definitive or tentative: $\mathrm{LR}=9.80, n=104$, $d f=1, P=0.002)$, and even more closely with RA EAC diagnoses (definitive according to ACR: $\mathrm{LR}=10.26$, $n=104, d f=1, P=0.001$, definitive or tentative: LR $=11.02, n=104, d f=1, P=0.001)$. Patient reported limitation of finger flexion and referral diagnoses were equivalent indicators for definitive RA diagnosis at EAC in binary logistic regression analysis. Only two parameters marginally exceeded these items in predicting RA: pathologic laboratory findings for one or more of the laboratory parameters (ESR, CRP, RF), and information about previous DMARD treatment. DMARD were prescribed before EAC appointment in eight RA patients, but these had already a disease duration of $>3$ years.

\section{Prediction of SpA and any IA}

Though inflammatory back pain reported by the doctor and the patient directed question on back pain at rest improving by exercise, however, showed significant agreement with each other $(\mathrm{LR}=37.5, n=99, d f=1, P<0.001)$, none of them could be correlated with EAC diagnosis of SpA. Composing answers on inflammatory back pain according to its more restrictive original definition [9] did not improve the predictive value for SpA to significant levels, but kept significant agreement with the patient directed question (LR $=19.5, n=102, d f=1, P<0.001)$. We were moreover unable to correlate $\mathrm{SpA}$ referral and EAC diagnosis.

HLA B27 status was reported for 20 patients in total, and for seven $\mathrm{SpA}$ patients (EAC diagnosis). The result was positive in eight and five patients, respectively. Calculated likelihood ratios for $\mathrm{SpA}$ diagnosis showed significance when including missing information in six field contingency tables (LR 24.99, $n=123, d f=2, P<0.001$ ), and also when excluding the patients without reported HLA B27 status (LR $=6.97, n=20, d f=1, P=0.008$ ). MRI of the pelvis or spine was provided in six, and gave significant evidence for EAC diagnosis in 4 of $14 \mathrm{SpA}$ cases (LR $=4.27, n=141, d f=1, P=0.039)$. None of the clinical items was in contrast predictive for $\mathrm{SpA}$ diagnosis at EAC.

Binary regression analysis for diagnosis of any inflammatory arthritis gave the best predictive values for reports on previous DMARD treatment and pathological findings in the basis laboratory analysis (ESR, CRP, RF), closely followed by information on limitations when clenching a fist and the referral diagnosis.

Questions on bodily function and previous treatment

Though answers on limited hand or finger function were informative for RA, more general questions on every day function gave no predictive information. The inability of leaving house was positively answered in only three patients, and the inability for professional activities was distributed among all tentiary or final diagnosis groups in 25 of 104 cases. 
Table 3 Answers on questions Q1, P1 and P4 of Table 1

\begin{tabular}{|c|c|c|c|c|}
\hline & Referral & $\%$ & EAC & $\%$ \\
\hline \multicolumn{5}{|l|}{ Q1 } \\
\hline Any joint swelling & \multicolumn{2}{|l|}{ n. s. } & \multicolumn{2}{|c|}{$\begin{aligned} \mathrm{LR} & =20.68, \mathrm{df}=7 \\
\mathrm{P} & =0.022\end{aligned}$} \\
\hline RA & $8 / 9$ & 88.9 & $11 / 11$ & 100 \\
\hline Suspected RA & $26 / 34$ & 76.5 & $5 / 6$ & 83.3 \\
\hline $\mathrm{SpA}$ & $1 / 1$ & 100 & $3 / 3$ & 100 \\
\hline Other infl. arthritides & $1 / 1$ & 100 & $8 / 9$ & 88.9 \\
\hline Other & $39 / 66$ & 59.1 & $48 / 82$ & 58.5 \\
\hline Joint swelling at hands or fingers & n.s. & & n.s. & \\
\hline RA & $4 / 8$ & 50.0 & $7 / 9$ & 77.8 \\
\hline Suspected RA & $21 / 25$ & 84.0 & $3 / 5$ & 60.0 \\
\hline $\mathrm{SpA}$ & $0 / 1$ & 0 & $0 / 0$ & \\
\hline Other infl. arthritides & $0 / 1$ & 0 & $4 / 7$ & 57.1 \\
\hline Other & $21 / 32$ & 65.6 & $32 / 43$ & 74.4 \\
\hline \multicolumn{5}{|l|}{$\mathrm{P} 1$} \\
\hline Any morning stiffness & n.s. & & n.s. & \\
\hline RA & $6 / 9$ & 66.7 & $8 / 10$ & 80.0 \\
\hline Suspected RA & $26 / 30$ & 86.7 & $4 / 6$ & 66.7 \\
\hline $\mathrm{SpA}$ & $1 / 1$ & 100 & $2 / 2$ & 100 \\
\hline Other infl. arthritides & $1 / 1$ & 100 & $8 / 11$ & 72.3 \\
\hline Other & $47 / 64$ & 73.4 & $59 / 76$ & 77.6 \\
\hline Morning stiffness $>30 \mathrm{~min}$ & \multicolumn{2}{|l|}{ n.s. } & \multicolumn{2}{|l|}{ n.s. } \\
\hline RA & $6 / 9$ & 66.7 & $8 / 10$ & 80 \\
\hline Suspected RA & $22 / 27$ & 81.5 & $3 / 6$ & 33.3 \\
\hline SpA & $1 / 1$ & 100 & $1 / 1$ & 100 \\
\hline Other infl. arthritides & $1 / 1$ & 100 & $8 / 11$ & 100 \\
\hline Other & $39 / 61$ & 63.9 & $49 / 71$ & 69.0 \\
\hline Morning stiffness $>60 \mathrm{~min}$ & \multicolumn{2}{|l|}{ n.s. } & \multicolumn{2}{|l|}{ n.s. } \\
\hline RA & $6 / 9$ & 66.7 & $8 / 10$ & 80 \\
\hline Suspected RA & $18 / 27$ & 66.6 & $2 / 6$ & 33.3 \\
\hline SpA & $1 / 1$ & 100 & $1 / 1$ & 100 \\
\hline Other infl. arthritides & $0 / 1$ & 0 & $8 / 11$ & 72.7 \\
\hline Other & $33 / 61$ & 54.1 & $42 / 71$ & 59.2 \\
\hline \multicolumn{5}{|l|}{ P4 } \\
\hline Incomplete clenching of a fist & \multicolumn{2}{|c|}{$\begin{aligned} \mathrm{LR} & =12.40, \mathrm{df}=4 \\
\mathrm{P} & =0.014\end{aligned}$} & \multicolumn{2}{|c|}{$\begin{aligned} \mathrm{LR} & =20.31, \mathrm{df}=7 \\
\mathrm{P} & =0.005\end{aligned}$} \\
\hline RA & $6 / 9$ & 66.6 & $5 / 7$ & 71.4 \\
\hline Suspected RA & $12 / 17$ & 70.6 & $3 / 6$ & 50 \\
\hline $\mathrm{OA}$ & & & $6 / 12$ & 50 \\
\hline $\mathrm{SpA}$ & $0 / 1$ & 0 & $1 / 2$ & 50 \\
\hline Other infl. arthritides & $0 / 1$ & 0 & $2 / 11$ & 18.2 \\
\hline Other & $12 / 65$ & 18.5 & $13 / 73$ & 17.8 \\
\hline
\end{tabular}

conditions ( 15.3 vs. $8.1 \%)$. The rates of monthly referrals in contrast, the proportions of referring medical specialists (general practitioners and internal medicine $61.8 \%$ before vs. $62.0 \%$ after introduction of the questionnaire, orthopaedic surgeons 23.6 vs. $29.0 \%$, other specialists 14.5 vs. $9.0 \%$, respectively), and the basis patient characteristics for age and gender remained stable. 


\section{Discussion}

This study shows that reliable information can be obtained for planning appointments at an EAC from questionnaires for referring doctors and patients. Even when considering all the necessary caution to be taken, however, a notable disagreement in the diagnosis of rheumatic conditions by rheumatologists and other disciplines became obvious. Our study suggests deficits and uncertainties among referring doctors especially for interpreting the patient history and clinical findings. In theory, this may be simply the result of a negative selection among colleagues realizing their limitations, which therefore decide to refer their patient to a rheumatology center. Nevertheless, our report is not a single observation [5], and is also supported by statements of a recent WHO publication [11]. It appears, however, necessary in the context to self critically acknowledge two third of referred patients before, but still about one third of them remaining with an uncertain diagnosis after first EAC appointment in our cohort when strictly following the classification criteria. This proportion was exactly in the range of a previous observation [12]. Suspected RA, however, could be confirmed or supported in 18 and excluded in 69 of 87 cases. Teaching definitely improves the diagnostic skills temporarily [13], but early onset RA and SpA is rather infrequent in GPs daily practice. We assume that this circumstance interferes with the maintenance of acquired skills. Our study clearly indicates that just reminding of items deduced from the RA or $\mathrm{SpA}$ classification criteria by using a questionnaire could not resolve existing problems in use.

As not the report of swollen hand or finger joints, but of any localization was valuable for EAC diagnosis of RA, we suggest major difficulties especially for the assessment of the carpal joint regions among inexperienced colleagues. Patient directed questions on limited grip function of digits 2-5 were in contrast predictive for RA, and the diagnostic value of this information was clearly superior to given information on morning stiffness. The most important confounding diagnosis when using the functional item was OA. Here, we should remind that pathologies of distal interphalangeal (DIP) joints, which are typically involved in idiopathic osteoarthritis, cause minor functional handicaps. These joints contribute little to the flexion of digits $2-$ 5 , and their functional decline could be completely compensated by intact proximal finger joints. RA in contrast typically affects the metacarpophalangeal (MCP), proximal interphalangeal (PIP), and the radiocarpal joints, which all are highly relevant for grip function. The overall functional consequences of these pathologic processes in RA seem to be quite accurately displayed by simple self-testing of clenching a fist. Tendon pathologies, however, could also limit the flexor function and occur in several conditions, thereby limiting higher disease specificity. Function can start to decline in RA already from a very early stage, and this observation is of value for predicting morbidity [14], mortality [15], and economic consequences [16]. Selfassessment of the swollen joint status even from experienced patients with average disease duration of 10 years appears far from being trustworthy [17]. We therefore disapprove self-assessment of swollen joints by patients referred to an EAC.

Pre-referral communication, either done by Fax, Email or otherwise electronically appears timely and appropriate for displaying functional limitations. Questionnaires can be an option for improving interdisciplinary communication and collaboration. Following our data, provided information about joint swelling of the hands by unskilled doctors must be handled with caution, and an authentic report of early SpA symptoms appears even more difficult. The use of technical parameters (HLA B27, MRI) is valuable for early SpA diagnosis, but these technical parameters are currently not recommended for use in unselected patients [2]. Nevertheless, these tools found already acceptance and were rather accurately applied in the meantime following our data also by non-rheumatologists.

Following our observations, major attention must be given to the possibility of unintended implications by treatment directed questionnaire items. We therefore primarily recommend, wherever triaging is necessary due to limited resources, a referral letter reporting the diagnosis, and previous diagnostic attempts and therapies only in empty plain text fields. A separate patient directed questionnaire focusing on specific functional limitations of RA and possibly also SpA appears useful and could be provided online [18, 19], which will probably improve the completion rate. As long as we strongly depend on the reliable clinical assessment of the swollen joint status, it appears that rheumatologists and specialized co-workers [13] have to ascertain the quality of this mean.

\section{References}

1. American College of Rheumatology subcommittee on rheumatoid arthritis guidelines (2002) Guidelines for the management of rheumatoid arthritis. Arthritis Rheum 46:328-346

2. Sieper J, Rudwaleit M (2005) Early referral recommendations for ankylosing spondylitis (including pre-radiographic and radiographic forms) in primary care. Ann Rheum Dis 64:659-663

3. Irvine S, Munro R, Porter D (1999) Early referral, diagnosis, and treatment of rheumatoid arthritis: evidence for changing medical practice. Ann Rheum Dis 58:510-513

4. Emery P, Breedveld FC, Dougados M, Kalden JR, Schiff MH, Smolen JS (2002) Early referral recommendation for newly diagnosed rheumatoid arthritis: evidence based development of a clinical guide. Ann Rheum Dis 61:290-297

5. Courtney PA, Wright GD (2001) Referrals to an "early synovitis clinic": are they appropriate? Ann Rheum Dis 60:991-992 
6. Schumacher HR, Pessler F, Chen LX (2003) Diagnosing early rheumatoid arthritis (RA). What are the problems and opportunities? Clin Exp Rheumatol 21(Suppl 31):S15-S19

7. Rat AC, Henegariu V, Boissier MC (2004) Do primary care physicians have a place in the management of rheumatoid arthritis? Joint Bone Spine 71:190-197

8. Arnett FC, Edworthy SM, Bloch DA, McShane DJ, Fries JF, Cooper NS et al (1988) The American rheumatism association 1987 revised criteria for the classification of rheumatoid arthritis. Arthritis Rheum 31:315-324

9. Calin A, Porta J, Fries JF, Schurman DJ (1977) Clinical history as a screening test for ankylosing spondylitis. JAMA 237:2613-2614

10. Dougados M, van der Linden S, Juhlin R, Huitfeldt B, Amor B, Calin A et al (1991) The European spondylarthropathy study group preliminary criteria for the classification of spondyloarthropathy. Arthritis Rheum 34:1218-1227

11. Akesson K, Dreinhofer KE, Woolf AD (2003) Improved education in musculoskeletal conditions is necessary for all doctors. Bull World Health Organ 81:677-678

12. van der Horst-Bruinsma IE, Speyer I, Visser H, Breedveld FC, Hazes JM (1998) Diagnosis and course of early-onset arthritis: results of a special early arthritis clinic compared to routine patient care. Br J Rheumatol 37:1084-1088

13. Gormley GJ, Steele WK, Gilliland A, Leggett P, Wright GD, Bell AL, Matthews C, Meenagh G, Wylie E, Mulligan R, Stevenson M, O'Reilly D, Taggart AJ (2003) Can diagnostic triage by general practitioners or rheumatology nurses improve the positive predictive value of referrals to early arthritis clinics? Rheumatology 42:763-768

14. Cohen JD, Dougados M, Goupille P, Cantagrel A, Meyer O, Sibilia J, Daures JP, Combe B (2006) Health assessment questionnaire score is the best predictor of 5-year quality of life in early rheumatoid arthritis. J Rheumatol 33:1936-1941

15. Farragher TM, Lunt M, Bunn DK, Silman AJ, Symmons DP (2007) Early functional disability predicts both all cause and cardiovascular mortality in people with inflammatory polyarthritis: results from the Norfolk Arthritis Register. Ann Rheum Dis 66(4):486-492

16. Kobelt G, Lindgren P, Lindroth Y, Jacobson L, Eberhardt K (2005) Modelling the effect of function and disease activity on costs and quality of life in rheumatoid arthritis. Rheumatology 44:1169-1175

17. Guillemin F, Saraux A, Fardellone P, Guggenbuhl P, Behier JM, Coste J (2003) Detection of cases of inflammatory rheumatic disorders: performance of a telephone questionnaire designed for use by patient interviewers. Ann Rheum Dis 62:957-963

18. Brophy S, Hunniford T, Taylor G, Menon A, Roussou T, Calin A (2004) Assessment of disease severity (in terms of function) using the internet. J Rheumatol 31:1819-1822

19. Ritter P, Lorig K, Laurent D, Matthews K (2004) Internet versus mailed questionnaires: a randomized comparison. J Med Internet Res 6:e29 